\title{
Natural Deduction for the Sheffer Stroke and Peirce's Arrow (And Any Other Truth-Functional Connective)
}

\author{
Richard Zach
}

the date of receipt and acceptance should be inserted later

\begin{abstract}
Methods available for the axiomatization of arbitrary finite-valued logics can be applied to obtain sound and complete intelim rules for all truth-functional connectives of classical logic including the Sheffer stroke (NAND) and Peirce's arrow (NOR). The restriction to a single conclusion in standard systems of natural deduction requires the introduction of additional rules to make the resulting systems complete; these rules are nevertheless still simple and correspond straightforwardly to the classical absurdity rule. Omitting these rules results in systems for intuitionistic versions of the connectives in question.
\end{abstract}

Mathematics Subject Classification (2010) 03A05, $03 \mathrm{~F} 03$

\section{Introduction}

In a recent paper, Hazen and Pelletier (2014) compared Jaśkowski's (1934) and Gentzen's (1934) versions of natural deduction. In Section 2.3 of their paper, Hazen and Pelletier consider Price's (1961) natural deduction rules for the Sheffer stroke (NAND). These rules are:

$$
\begin{aligned}
& {[A]} \\
& \vdots \\
& \frac{B \mid B}{A \mid B}\left|I_{P} \quad \frac{A \quad A \mid B}{B \mid B}\right| E_{P} \quad \frac{A \quad(B \mid B) \mid A}{B} \| E_{P}
\end{aligned}
$$

Although these rules are sound and complete for a logic in which $\mid$ is the only primitive, they are not of the standard "intelim" form. Hazen and Pelletier attempt to give a JaśkowskiFitch style natural deduction system in Section 3.3 which comes closer to this ideal but find that their rules do not characterize the classical logic of $\mid$ except in the attenuated sense that adding these rules to a standard natural deduction system allows one to prove $(A \mid B) \leftrightarrow$ $\neg(A \wedge B)$ ("parasitic completeness"). By themselves, their rules are "ambiguous" between the two intuitionistic versions $\neg(A \wedge B)$ and $\neg A \vee \neg B$. They then discuss the framework

Published in: Journal of Philosophical Logic (2016) 45:183-197. DOI 10.1007/s10992-015-9370-x

Richard Zach

University of Calgary, Department of Philosophy, 2500 University Dr NW, Calgary, AB T2N0A9, Canada, http://ucalgary.ca/rzach/, E-mail: rzach@ucalgary.ca 
of Schröder-Heister's generalized natural deduction and show that Došen's $\star$, which is an "indigenous Sheffer function" for intuitionistic logic in the sense that every connective can be expressed in terms of it, can be given intelim rules in this framework. They leave open the question of how the classical Sheffer stroke should be dealt with in natural deduction, as well as the status of their proposed rules. The aim of this note is to show how one can give a natural deduction system of standard intelim rules for the classical Sheffer stroke and its intuitionistic variants, and to discuss the properties of the resulting systems. It is of course possible to arrive at the same rules directly; indeed, Read (1999) did so. The methods presented here, however, are completely general and can be applied to any set of truth-functional connectives; we sketch this also for Pierce's arrow (NOR) and exclusive or.

\section{Obtaining natural deduction rules}

In a series of papers, Baaz et al (1993, 1994); Zach (1993) showed how finite-valued logics can be given sequent calculus axiomatizations based on a simple procedure that yields introduction rules for each connective and truth value, and where sequents have as many places as the logic has truth values. They proved completeness, cut-elimination, midsequent theorem, interpolation, and generalized it to multiple-conclusion natural deduction systems for finite-valued logics. The natural deduction systems have the normal form property, and there are translations from natural deduction derivations to sequent calculus proofs and vice versa. In the case of two-valued classical logic, the construction yields exactly the classical sequent calculus $\mathbf{L K}$, and a multiple-conclusion system of natural deduction with the standard introduction rules for $\neg, \wedge, \vee, \rightarrow, \forall$, and $\exists$, and "general elimination rules". This system is complete for classical logic without the need to add axioms (e.g., $A \vee \neg A$ ) or rules such as double negation elimination or Prawitz's $\perp_{C}$ to Gentzen's NJ. ${ }^{1}$

For the Sheffer stroke, this procedure yields sequent calculus rules as follows. The Sheffer stroke is a truth-functional connective, and any truth function can be expressed as a conjunctive normal form of its arguments. We can thus express the conditions under which a formula of the form $A \mid B$ is true in terms of a conjunction of disjunctions of $A, \neg A, B$, and $\neg B$. In the same way we can express the conditions under which $A \mid B$ is false, i.e., express $\neg(A \mid B)$ as a conjunctive normal form.

T $A \mid B$ is true iff $A$ is false or $B$ is false:

$$
A \mid B \Leftrightarrow \neg A \vee \neg B
$$

F $A \mid B$ is false iff $A$ is true and $B$ is true:

$$
\neg(A \mid B) \Leftrightarrow A \wedge B
$$

From these normal forms we then directly obtain introduction rules for $\mid$ in the succedent and antecedent. The normal form for $A \mid B$ provides the introduction rule for $\mid$ in the succedent $\mid \mathrm{R}$; that for $\neg(A \mid B)$ the introduction rule for the antecedent $\mid \mathrm{L}$. In each case, the conjuncts in the normal form correspond to the premises. In each premise sequent, put the formula $A$ on the right if it occurs without negation in the corresponding conjunct, and on

\footnotetext{
1 The general elimination rules coincide with the elimination rules of Parigot's (1992a) free deduction; he showed how to obtain the standard elimination rules from them by systematic simplification. A version of the simplified system of classical multiple-conclusion natural deduction was studied by Parigot (1992b). The general elimination rules for $\wedge$ and $\rightarrow$ were studied by von Plato (2001).
} 
the left if it occurs negated, and the same for $B$. Thus, for $\mid R$ we have one premise since the conjunctive normal form has just one conjunct $\neg A \vee \neg B$, and since $A$ and $B$ are both negated, $A$ and $B$ both go on the left: $A, B \vdash$. For $\mid L$ we have two premises corresponding the the two (one-element) disjunctions $A$ and $B$ : $\vdash A$ and $\vdash B$. The sequents in the full rule then also contain the context formulas $\Gamma, \Delta$.

$$
\frac{A, B, \Gamma \vdash \Delta}{\Gamma \vdash \Delta, A \mid B}\left|R \quad \frac{\Gamma \vdash \Delta, A \quad \Gamma \vdash \Delta, B}{A \mid B, \Gamma \vdash \Delta}\right| L
$$

Let us call the sequent calculus that includes these two rules, the axioms $A \vdash A$, and the usual structural rules (weakening, exchange, contraction on the left and right, as well as cut) LS.

Proposition 1 LS is sound and cut-free complete.

Proof Follows as a special case of Baaz et al (1994, Theorems 3.1, 3.2) and Zach (1993, Theorems 3.3.3, 3.3.10).

Gentzen-style natural deduction rules are obtained from sequent calculus rules by turning the premises "sideways." Formulas in the antecedent of a premise become assumptions. The auxiliary formulas in the antecedent are those that may be discharged in an application of the rule; the context formulas $\Gamma$ are suppressed. Formulas in the consequent become the conclusion of the corresponding premise. For the introduction rules, the premises then are just the sideways premises of the $\mid \mathrm{R}$ rule. For the elimination rule, the principal formula $A \mid B$ in the $\mid L$ rule becomes an additional premise for the $\mid E$ rule. The natural deduction rules for $\mid$ obtained this way then are:

$$
\begin{aligned}
& {[A]:[B]} \\
& \frac{\dot{\Delta}}{\Delta, A \mid B}\left|I_{m} \quad \frac{\Delta, A \mid B \quad \Delta, A \quad \Delta, B}{\Delta}\right| E_{m}
\end{aligned}
$$

Note that this version of natural deduction allows multiple formulas in the conclusion position. Let us refer to this system as $\mathbf{N S}_{m}$, the subscript $m$ for "multiple conclusion." It requires structural rules that allow conclusions to be weakened, contracted, and rearranged. As for all the natural deduction systems constructed in this way, there are simple translations of proofs in $\mathbf{L S}$ into deductions in $\mathbf{N S} \mathbf{S}_{m}$ and vice versa. The system $\mathbf{N} \mathbf{S}_{m}$ is thus also sound and complete for the classical Sheffer stroke.

Proposition 2 LS proves a sequent $\Gamma \vdash \Delta$ iff $\mathbf{N S}_{m}$ derives $\Delta$ from assumptions $\Gamma^{\prime}$ with $\Gamma^{\prime} \subseteq \Gamma$.

Proof Follows from the general results in Baaz et al (1993, Theorem 4.7, 5.4) and Zach (1993, Theorem 4.2.8, 4.3.4). See Prop. 3 below for the translation in this specific case.

\section{Single-conclusion natural deduction}

Natural deduction systems commonly require that each conclusion is a single formula. In order to obtain rules for a single-conclusion natural deduction system, we begin by introducing a corresponding restriction into the sequent calculus: the succedent in each sequent 
in a proof must contain at most one formula. This in effect means that the $|L|$,$R , and W R$ (weakening-right) rules become:

$$
\frac{A, B, \Gamma \vdash}{\Gamma \vdash A \mid B}\left|R^{\prime} \quad \frac{\Gamma \vdash A \quad \Gamma \vdash B}{A \mid B, \Gamma \vdash}\right| L^{\prime} \quad \frac{\Gamma \vdash}{\Gamma \vdash A} W R^{\prime}
$$

Exchange and contraction on the right are no longer needed. We will refer to the resulting calculus as $\mathbf{L} \mathbf{S}^{\prime}$.

For the corresponding natural deduction system, we also have to accommodate the empty sequence of conclusion formulas, i.e., we have to introduce a placeholder symbol $\perp$ for the empty conclusion in proofs. The inference rules will be adapted for single conclusions by replacing empty conclusions sequences by $\perp$. The single conclusion rules corresponding to $\left|R^{\prime},\right| L^{\prime}$, and $W R^{\prime}$ then are:

$$
\begin{aligned}
& {[A] \quad[B]} \\
& \vdots \\
& \frac{\dot{\perp}}{A \mid B}\left|I \quad \frac{A \mid B \quad A \quad B}{\perp}\right| E \quad \frac{\perp}{A} \perp_{I}
\end{aligned}
$$

The $\perp_{I}$ rule is named as in (Prawitz 1965); Gentzen (1934) did not give a name to the rule. The special cases where $A=B$ correspond to Gentzen's $\neg I$ and $\neg E$ rules:

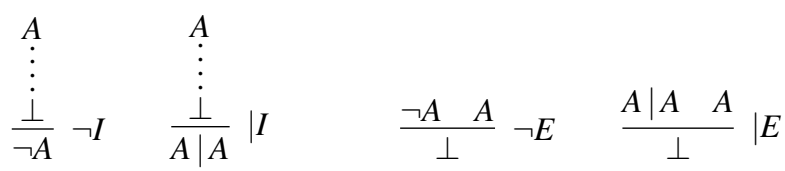

The resulting system NS exactly corresponds to $\mathbf{L} \mathbf{S}^{\prime}$, the sequent calculus where the succedent of each sequent is restricted to at most one formula. ${ }^{2}$

Proposition 3 NS derives A from assumptions in $\Gamma$ iff $\mathbf{L} \mathbf{S}^{\prime}$ proves the sequent $\Gamma \vdash A$ (or $\Gamma \vdash$ if $A=\perp$ ).

Proof We translate each proof in $\mathbf{L} \mathbf{S}^{\prime}$ of $\Gamma \vdash A$ into a deduction in NS of $A$, and one of $\Gamma \vdash$ into one of $\perp$, where all open assumptions are in $\Gamma$. Conversely, any deduction in NS of $A$ from assumptions $\Gamma$ can be translated into a proof in $\mathbf{L} \mathbf{S}^{\prime}$ of $\Gamma \vdash A$, or of $\Gamma \vdash$ if $A=\perp$.

Assume LS proves $\Gamma \vdash A$ or (or $\Gamma \vdash$ ) via a proof $\pi$. We construct an NS derivation $\delta$ of $A$ (or $\perp$ ) from assumptions $\Gamma^{\prime} \subseteq \Gamma$ by induction. If $\pi$ only consists of the an axiom $A \vdash A$, then $A$ is a derivation of $A$ (which depends on $A$ ). If $\pi$ ends in a $W R$, the conclusion is $\Gamma \vdash A$ and the premise is $\Gamma \vdash$. By induction hypothesis, we have a derivation of $\perp$ from $\Gamma^{\prime}$. Add $\perp_{I}$ with conclusion $A$ to this derivation.

If $\pi$ ends in a cut, the last inference is of the form

$$
\frac{\Gamma_{1} \vdash A \quad A, \Gamma_{2} \vdash B}{\Gamma_{1}, \Gamma_{2} \vdash B} c u t
$$

2 The use of the special symbol $\perp$ is not strictly necessary, as it could be replaced by explicitly contradictory premises (e.g., $C \mid D, C$, and $D$; or more simply $C \mid C$ and $C$ ) when it appears as a premise, and by an arbitrary formula when it appears as the conclusion. This has the advantage that the resulting rules mention no symbols other than |, i.e., are pure. Indeed, this was in part Hazen and Pelletier's goal and their rules used this approach. This alternative approach can however not be used if no such "explicit contradiction" can be expressed. See also Section 7 below. 
By induction hypothesis, we have NS derivations $\delta_{1}$ of $A$ from assumptions in $\Gamma_{1}$ and a derivation of $B$ from assumptions in $A, \Gamma_{2}$.

If $A$ is not actually an open assumption in $\delta_{2}, \delta_{2}$ already is a derivation of $B$ from $\Gamma_{2}^{\prime} \subseteq \Gamma_{1} \cup \Gamma_{2}$. Otherwise, $\delta_{2}$ does contain undischarged assumptions $A$. Append the derivation $\delta_{1}$ of $A$ (with open assumptions $\Gamma_{1}^{\prime} \subseteq \Gamma_{1}$ ) to all leaves where $A$ is undischarged in $\delta_{2}$. This is a derivation of $B$ from assumptions $\Gamma_{1}^{\prime}, \Gamma_{2}^{\prime} \subseteq \Gamma_{1}, \Gamma_{s}$.

If $\pi$ ends in $\mid R^{\prime}$, the last sequent is $\Gamma \vdash A \mid B$ and the premise is $A, B, \Gamma \vdash$. By induction hypothesis there is a derivation $\delta_{1}$ of $\perp$ with open assumptions among $A, B, \Gamma$. Add a $\mid I$ rule to $\delta_{1}$ and discharge any assumptions of the form $A$ or $B$.

If $\pi$ ends in $\mid L$, the last sequent is $A \mid B, \Gamma \vdash$ and the premises are $\Gamma \vdash A$ and $\Gamma \vdash B$. By induction hypothesis we have deductions $\delta_{1}$ of $A$ from $\Gamma_{1} \subseteq \Gamma$ and $\delta_{2}$ of $B$ from $\Gamma_{2} \subseteq \Gamma$, respectively. Use these and an assumption $A \mid B$ as premises for a $\mid E$ rule with conclusion $\perp$. The result is a derivation of $\perp$ from assumptions $A\left|B, \Gamma_{1}, \Gamma_{2} \subseteq A\right| B, \Gamma$.

In the other direction we proceed the same way. Derivations consisting of assumptions $A$ alone are translated into axiom sequents $A \vdash A$. Derivations ending in $\perp_{I}$ result in proofs ending in $W R$. If a derivation ends in $\mid I$ with the premise $\perp$ being derived from assumptions $A, B, \Gamma$, by induction hypothesis we have an $\mathbf{L} \mathbf{S}^{\prime}$-proof of $A, B, \Gamma \vdash$. Apply $\mid R$ to obtain a proof of $\Gamma \vdash A \mid B$. If a derivation ends in $\mid E$, its premises are $A \mid B, A$, and $B$ and its conclusion is $\perp$. By induction hypothesis, we have $\mathbf{N} \mathbf{S}^{\prime}$-proofs $\pi_{1}, \pi_{2}, \pi_{3}$ of $\Gamma_{1} \vdash A \mid B, \Gamma_{2} \vdash A$ and $\Gamma_{3} \vdash B$ with $\Gamma_{i} \cup \Gamma_{2} \cup \Gamma_{3}=\Gamma$. We obtain a proof of $\Gamma \vdash \Delta$ as follows:

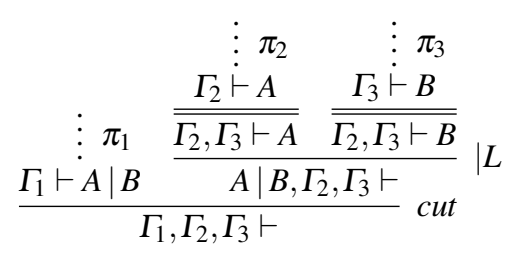

\section{Intuitionistic Sheffer strokes}

It is easily seen that the systems $\mathbf{L} \mathbf{S}^{\prime}$ and $\mathbf{N S}$ are not complete. Consider a three-valued interpretation with truth values $\perp<I<\top$ with $\top$ designated, and consider the following truth table for |:

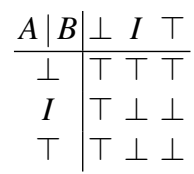

$\mathbf{L S} \mathbf{S}^{\prime}$ is sound for this interpretation, i.e., whenever $\mathbf{L} \mathbf{S}^{\prime}$ proves $\Gamma \vdash A$ then $\min \{v(A): A \in$ $\Gamma\} \leq v(A)$, and whenever it proves $\Gamma \vdash$, then $\min \{v(A): A \in \Gamma\}=\perp$, for any truth-value assignment $v$. Consider $(A \mid A) \mid(A \mid A)$. For the assignment $v(A)=I$, we have $v((A \mid A) \mid(A \mid$ $A))=\top>v(A)$, hence the sequent $(A \mid A) \mid(A \mid A) \vdash A$ is unprovable in $\mathbf{L} \mathbf{S}^{\prime}$. Consequently there is no NS deduction of $A$ from the only assumption $(A \mid A) \mid(A \mid A)$. This should not be surprising. If we abbreviate $A \mid A$ as $\neg A$, then this amounts to the intuitionistically invalid inference of double negation elimination, i.e., $\neg \neg A \vdash A$.

Since $\mathbf{L} \mathbf{S}^{\prime}$ and $\mathbf{N S}$ are not complete for the Sheffer stroke the question arises if one can give a semantics for $\mid$ relative to which these systems are complete. Indeed this is the case. It 
is again not surprising that a version of intuitionistic Kripke frames can serve this purpose. This can be obtained by applying a general result about all finite-valued logics due to Baaz and Fermüller (1996) to the two-valued case and the truth table for $\mid$. An intuitionistic Kripke model is $M=\langle W, \leq, v\rangle$ where $W$ is a non-empty set of worlds, $\leq$ is a partial order on $W$, and $v$ is a function from the propositional variables to $\wp(W)$ satisfying $p \in v\left(w^{\prime}\right)$ whenever $w \leq w^{\prime}$ and $p \in v(w)$ (in other words, the interpretation of predicate variables is monotonic). We then define the satisfaction relation by:

1. $M, w \Vdash p$ iff $v(p) \in w$

2. $M, w \Vdash A \mid B$ iff for all $w^{\prime} \geq w$, either $M, w^{\prime} \nVdash A$ or $M, w^{\prime} \nVdash B$.

(If one wants to also include other connectives $\neg, \vee, \wedge$, etc., the corresponding standard satisfaction conditions for them may be added as well. It is then evident that $M, w \Vdash A \mid B$ iff $M, w \Vdash \neg(A \wedge B)$ as one would expect.) One can then easily verify soundness, and prove the following completeness theorem by a version of Schütte's reduction tree method:

Proposition 4 If $\mathbf{L} \mathbf{S}^{\prime}$ does not prove $\Gamma \vdash A$, then there is a Kripke model $M$ and a world $w$ so that $M, w \Vdash B$ for all $B \in \Gamma$ but $M, w \nVdash A$.

Proof See Baaz and Fermüller (1996, Theorem 4.2).

If the rules $|I|$,$E are added to \mathbf{N J}$ instead of being considered on their own, we can also show completeness by proving that $A \mid B \vdash \neg(A \wedge B)$ and $\neg(A \wedge B) \vdash A \mid B$. This is what Hazen and Pelletier call "parasitic completeness," but it has one interesting consequence: it shows that our rules for $\mid$ in LS (and hence also the intelim rules in NS) are-unlike their rulesnot ambiguous between two different connectives: in LS and NS, | defines the intuitionistic connective $\neg(A \wedge B)$.

This of course raises the question: what $d o$ Hazen and Pelletier's rules define, if anything?

Let us use $\|$ for the intuitionistic connective introduced by Hazen and Pelletier. Their rules are formulated for a Jaśkowski-Fitch style natural deduction system in which proofs (and subproofs) are sequences of formulas with scope of assumptions indicated, and instead of a primitive $\perp$ they make use of "explicit contradictions," i.e., triples of formulas of the forms $A \mid B, A, B$. In our Gentzen-style framework, their rules can be formulated as follows:

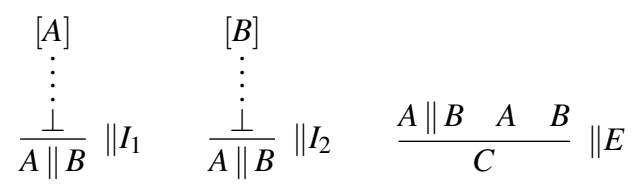

These rules correspond to the intuitionistic sequent rules:

$$
\frac{A, \Gamma \vdash}{\Gamma \vdash A \| B}\left\|R_{1}^{\prime} \quad \frac{B, \Gamma \vdash}{\Gamma \vdash A \| B}\right\| R_{2}^{\prime} \quad \frac{\Gamma \vdash A \quad \Gamma \vdash B}{A \| B, \Gamma \vdash} \| L^{\prime}
$$

The versions $\left\|R_{1},\right\| R_{2}, \| L$ of these rules without the restriction to a single formula in the succedent are of course equivalent to $\mid L$ and $\mid R$, i.e., in the presence of contraction on the right, LS plus $\left\|R_{1},\right\| R_{2}, \| L$ proves both $(A \mid B) \vdash(A \| B)$ and $(A \| B) \vdash(A \mid B)$. In the intuitionistic system, i.e., $\mathbf{L} \mathbf{S}^{\prime}$ plus $\| R_{1}^{\prime}$, $\| R_{2}^{\prime}$, $\|_{L}^{\prime}$, only $(A \| B) \vdash(A \mid B)$ is provable. This corresponds to the provability in $\mathbf{L} \mathbf{J}$ of $(\neg A \vee \neg B) \vdash \neg(A \wedge B)$ and the unprovability of $\neg(A \wedge B) \vdash(\neg A \vee \neg B)$. 
However, while $\mid$ defined by $\left|R^{\prime},\right| L^{\prime}$ characterizes $A \mid B$ intuitionistically as $\neg(A \wedge B)$, $\left\|R_{1}^{\prime},\right\| R_{2}^{\prime}, \| L^{\prime}$ do not, as one might at first glance suspect, intuitionistically define $A \| B$ as $\neg A \vee \neg B$. In other words, $\left\|R_{1}^{\prime},\right\| R_{2}^{\prime}, \| L^{\prime}$ is not parasitically complete: $\mathbf{L J}$ plus these rules does not prove $(A \| B) \vdash(\neg A \vee \neg B)$. (This can be shown by appealing to Harrop's (1960) extended version of the disjunction property in $\mathbf{L} \mathbf{J}$; see Takeuti 1987, Theorem 6.14 or Troelstra and Schwichtenberg 2000, Theorem 4.2.3.) Note also that the rules do not suffice to even prove $A\|B \vdash A\| B$ from axioms $A \vdash A$ and $B \vdash B$.

In order to complete the calculus so as to obtain these results, one could replace the $\| L$ rule with the following two:

$$
\frac{A\|A, \Gamma \vdash C \quad B\| B, \Gamma \vdash C}{A \| B, \Gamma \vdash C} \| L^{\prime \prime} \quad \frac{\Gamma \vdash A}{A \| A, \Gamma \vdash} \neg R
$$

The corresponding natural deduction rules are: ${ }^{3}$

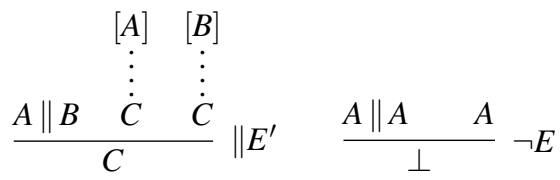

\section{Classical single-conclusion systems}

We have seen that the intelim rules for $\mid$ do not by themselves yield a complete natural deduction system for the classical Sheffer stroke. To obtain a classically complete system we have to add a new rule $\mid E_{C}$ which corresponds to the classical $\neg E$ rule (Prawitz's $\perp_{C}$ ).

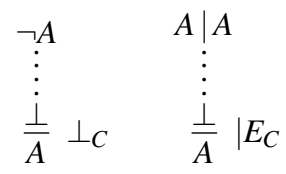

This results in a system $\mathbf{N S}_{C}$ complete for the classical interpretation of $\mid$. For instance, we can deduce $A$ from $(A \mid A) \mid(A \mid A)$ as follows:

$$
\frac{(A \mid A) \mid(A \mid A)[A \mid A]^{1}}{\frac{\perp}{A} \mid E_{C}: 1} \mid E
$$

Proposition $5 \mathbf{N S}_{C}$ is sound and complete.

Proof Completeness is proved by extending the translation between $\mathbf{L S}^{\prime}$-proofs and NSdeductions of Prop 3 to translations between $\mathbf{L} \mathbf{S}_{C}^{\prime}$-proofs and $\mathbf{N S} \mathbf{S}_{C}$-deductions, where $\mathbf{L} \mathbf{S}_{C}^{\prime}$ is the calculus resulting from $\mathbf{L} \mathbf{S}^{\prime}$ by adding the rule

$$
\frac{\neg A, \Gamma \vdash A}{\Gamma \vdash A} \mid L_{C}
$$

One then also shows, by induction on the height of proofs, that if $\mathbf{L S}_{C}^{\prime}$ proves $\Gamma, \neg \Delta \vdash A$ then $\mathbf{L S}$ proves $\Gamma \vdash \Delta, A$, and conversely, if $\mathbf{L S}$ proves $\Gamma \vdash \Delta, \Delta^{\prime}$ then $\mathbf{L S}_{C}^{\prime}$ proves $\Gamma, \neg \Delta \vdash \Delta^{\prime}$, where $\Delta^{\prime}$ may be empty or a single formula. This establishes that $\mathbf{L} \mathbf{S}_{C}^{\prime}$ is complete. (See also Troelstra and Schwichtenberg 2000, Section 3.3.2; our rule $\mid L_{C}$ plays the role of stability axioms here.)

\footnotetext{
3 See errata.
} 


\section{Normalization}

One important fact about Gentzen's natural deduction calculus NJ is that it normalizes, i.e., every derivation can be transformed into one in which is normal: no formula occurrence is both the conclusion of an introduction rule and the major premise of an elimination rule. Such formula occurrences are called maximal formulas. This result was first established in print by Prawitz (1965). It is proved by showing that maximal formula occurrences can be eliminated from derivations, one at a time. The result then follows by induction on the number of maximal formula occurrences in a derivation.

The result also holds for NS, and indeed also for $\mathbf{N J}$ to which we add the $\mid I$ and $\mid E$ rules. All that is required is to provide the reduction for maximal formulas of the form $A \mid B$; the argument of Prawitz (1965, Ch. II) otherwise goes through without change. If a formula occurrence of the form $A \mid B$ is maximal in a derivation, the derivation has the form on the left, and can be transformed into the derivation on the right, in which the maximal formula occurrence is removed:

$$
\begin{aligned}
& \text { [A] }[B]
\end{aligned}
$$

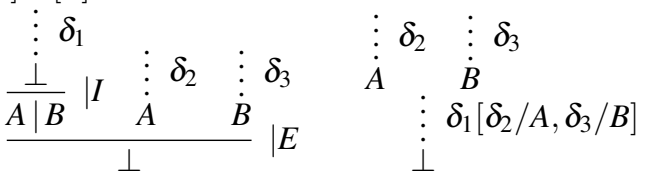

The derivation $\delta_{1}\left[\delta_{2} / A, \delta_{3} / B\right]$ is the derivation resulting from $\delta_{1}$ by appending $\delta_{2}$ to all assumptions of the form $A$ discharged by the $\mid I$ rule, and $\delta_{3}$ to all such assumptions of the form $B$.

Prawitz (1965, Ch. III) also showed that the classical system with intelim rules for $\neg$, $\wedge, \rightarrow, \forall$, with $\perp_{C}$, but without $\vee$ and $\exists$ also has normalization. Prawitz shows that for this system, we may restrict applications of $\perp_{C}$ to atomic formulas $A$. This is done by showing that any application of $\perp_{C}$ for a composite formula can be transformed into one where $\perp_{C}$ is instead applied to its immediate subformulas. The result then follows by induction on the complexity of formulas $A$ used in $\perp_{C}$ inferences. It is this step that does not work for formulas of the form $A \vee B$ or $\exists x A(x)$.

We may extend Prawitz's result to $\mathbf{N S}_{C}$ and to natural deduction systems which include $\neg$ and its rules including $\perp_{C}$ directly, or which define $\neg A$ as $A \mid A$ and use the rules for |, including $\mid E_{C}$. To do this, it suffices to show that the fact mentioned above (i.e., Prawitz's Th. 1 of Ch. III) also holds for NS and related systems not including $\vee$. An application of $\mid E_{C}$ applied to $A \mid B$ in a derivation appears as the subderivation on the left, which can be replaced by the subderivation on the right (for simplicity, abbreviate $C \mid C$ by $\neg C$ ):

$$
\begin{array}{cc} 
& \frac{[A \mid B]^{1} \quad[A]^{2}}{}[B]^{2} \\
{[\neg(A \mid B)]^{1}} & \frac{\perp}{\neg(A \mid B)} \mid I: 1 \\
\vdots \delta & \vdots \delta^{\prime} \\
\frac{\perp}{A \mid B} \mid E_{C}: 1 & \frac{\perp}{A \mid B} \mid I: 2
\end{array}
$$

In $\delta^{\prime}$, the assumptions in $\delta$ of the form $\neg(A \mid B)$ which are discharged by the $\mid E_{C}$ rule are replaced by the derivations of $\neg(A \mid B)$ indicated. 


\section{Jaśkowski-Fitch and Suppes-Lemmon natural deduction}

The natural deduction systems of Jaśkowski (1934) and Fitch (1952) and their variants are not commonly used for proof-theoretic investigations, but they are the most common systems of natural deduction appearing in introductory textbooks. They differ from Gentzenstyle single-conclusion natural deduction systems in a number of ways: First, while in Gentzen-type systems proofs have a tree structure, in Jaśkowski-Fitch style systems they are linear sequences of formulas, which may however be grouped into possibly nested subproofs. Every subproof begins with an assumption, and subproofs themselves can serve as premises to inferences. The conclusion of these inferences then no longer depend on the assumption of the subproof.

In order to obtain a Jaśkowski-Fitch type system for the Sheffer stroke, specifically, to accommodate the $\mid I$ rule, we must allow for subproofs to have two assumption formulas, or alternatively, relax the restriction on subproofs having their last formula not appear in a nested subproof itself. The corresponding versions of the $|I|$,$E , and \mid E_{C}$ rules are as follows: ${ }^{4}$

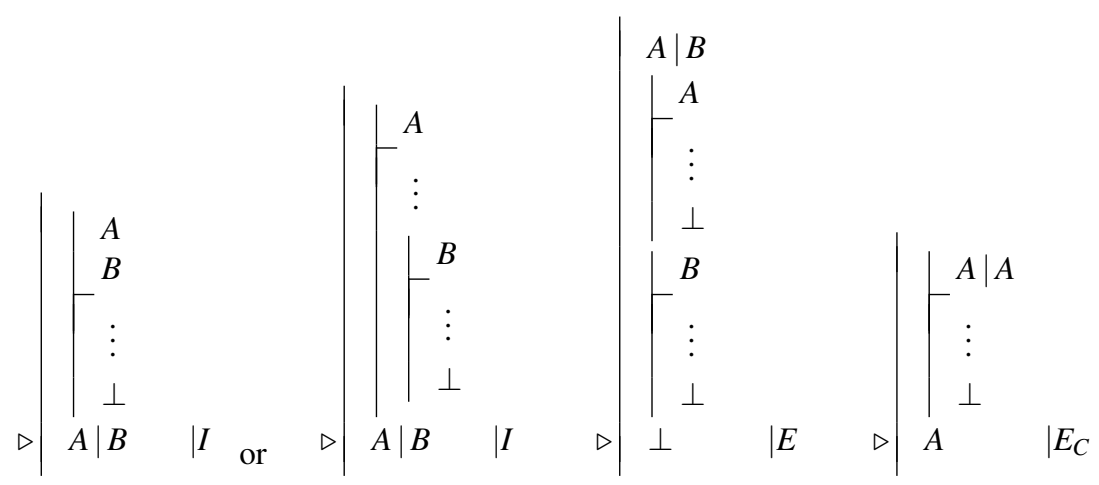

Some presentations of Fitch-style systems lack the $\perp$ constant as a primitive, and instead require the presence of an "explicit contradiction," i.e., both $A$ and $\neg A$, when $\perp$ appears as a premise or the last line of a subproof used as a premise. Instead of deriving $\perp$ as a conclusion, the corresponding rules allow the derivation of an arbitrary formula. Rules which result in such a system (or which can be added to such a system) would be:

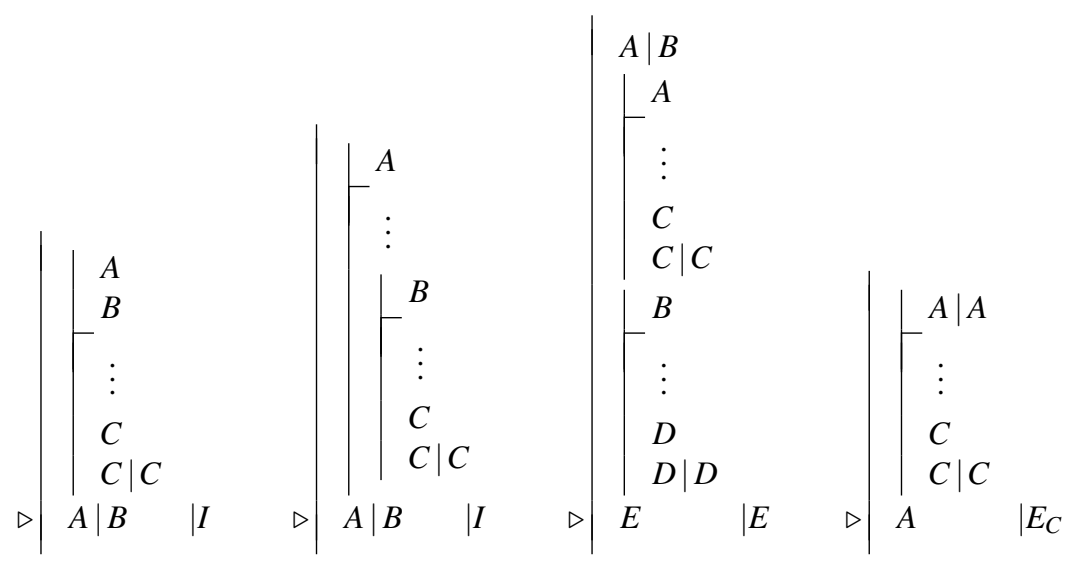

\footnotetext{
4 The $\mid E$ rules below do not correspond to the natural deduction rules given above. See errata section at
} end. 
No relaxation of the syntax is needed in the style of natural deduction due to Suppes (1957) and Lemmon 1965, where the assumptions on which a formula depends are recorded in the deduction with the formulas themselves, and discharging of assumptions is done by allowing formulas to be removed from the assumption list. The rules might be stated as follows, following Lemmon:

|-Introduction: Given a proof of $\perp$ from $A$ and $B$ as assumptions, we may derive $A \mid B$ as conclusion. The conclusion depends on any assumptions on which $\perp$ depends in its derivation from $A$ and $B$ (apart from $A$ and $B$ ).

|-Elimination: Given a proof of $A \mid B$, together with a proof of $\perp$ from $A$ as assumption and a proof of $\perp$ from $B$ as assumption, we may derive $\perp$ as conclusion. The conclusion $\perp$ depends on any assumptions on which $A \mid B$ depends, or on which $\perp$ depends in its derivation from $A$ (apart from $A$ ), or on which $\perp$ depends in its derivation from $B$ (apart from $B A$ ).

Classical |-Elimination: Given a proof of $\perp$ from $A \mid A$ as assumption, we may derive $A$ as conclusion. $A$ depends on any assumptions on which $\perp$ depends in its derivation from $A \mid A$ (apart from $A \mid A$ ).

In these rules we may consider $\perp$ primitive, or read it as "a contradiction," which again might mean "both $C$ and $C \mid C$ " if no negation is available.

\section{Other connectives}

It bears emphasizing that the methods by which we have obtained the rules for $\mid$ are completely general and will produce rules for any other truth-functional connective with the same systematic features. For instance, Pierce's arrow $\downarrow$ or NOR, the dual of the Sheffer stroke, would be characterized by

$\mathrm{T} A \downarrow B$ is true iff $A$ is false and $B$ is false

$\mathrm{F} A \downarrow B$ is false iff $A$ is true or $B$ is true

which would result in the sequent rules

$$
\frac{A, \Gamma \vdash \Delta \quad B, \Gamma \vdash \Delta}{\Gamma \vdash \Delta, A \downarrow B} \downarrow R \quad \frac{\Gamma \vdash \Delta, A, B}{A \downarrow B, \Gamma \vdash \Delta} \downarrow L
$$

In order to obtain a single-conclusion sequent system we again leave out the side formulas $\Delta$. The presence of two formulas on the right in the premise of $\downarrow \mathrm{L}$ is an obstacle for turning this rule into single-conclusion natural deduction rule. However, we can replace $\downarrow L$ by two rules in which the auxiliary formulas $A$ and $B$ appear in the premise only once. By restricting the succedents to at most one formula, we obtain the following rules:

$$
\frac{A, \Gamma \vdash B, \Gamma \vdash}{\Gamma \vdash A \downarrow B} \downarrow R \quad \frac{\Gamma \vdash A}{A \downarrow B, \Gamma \vdash} \downarrow L_{1} \quad \frac{\Gamma \vdash B}{A \downarrow B, \Gamma \vdash} \downarrow L_{2}
$$

We obtain the single-conclusion natural deduction rules

$$
\begin{array}{cc}
{[A]} & {[B]} \\
\vdots & \vdots \\
\frac{\perp}{A \downarrow B} & \perp
\end{array} \quad \frac{A \downarrow B \quad A}{\perp} \downarrow E_{1} \quad \frac{A \downarrow B \quad B}{\perp} \downarrow E_{2}
$$


This natural deduction system, as well as the restricted sequent calculus, are again not classically complete, but are complete for an intuitionistic version of $\downarrow$. They can be made complete for classical $\downarrow$ by adding

$$
\begin{aligned}
& A \downarrow A \\
& \vdots \\
& \frac{\perp}{A} \downarrow E_{C}
\end{aligned}
$$

A slightly more complex example would be the XOR connective. Its truth and falsity conditions are given by:

T $A \oplus B$ is true iff ( $A$ is true or $B$ is true) and ( $A$ is false or $B$ is false)

F $A \oplus B$ is false iff ( $A$ is false or $B$ is true) and (B is false or $A$ is true)

The corresponding sequent rules are

$$
\frac{\Gamma \vdash \Delta, A, B \quad A, B, \Gamma \vdash \Delta}{\Gamma \vdash \Delta, A \oplus B} \oplus R \quad \frac{A, \Gamma \vdash \Delta, B \quad B, \Gamma \vdash \Delta, A}{A \oplus B, \Gamma \vdash \Delta .} \oplus L
$$

Again, the $\oplus \mathrm{R}$ rule may be replaced by the two rules

$$
\frac{\Gamma \vdash \Delta, A \quad A, B, \Gamma \vdash \Delta}{\Gamma \vdash \Delta, A \oplus B} \oplus R_{1} \quad \frac{\Gamma \vdash \Delta, B \quad A, B, \Gamma \vdash \Delta}{\Gamma \vdash \Delta, A \oplus B} \oplus R_{2}
$$

which result in the natural deduction rules

$$
\begin{array}{ccccc} 
& {[A],[B]} & {[A],[B]} & {[A]} & {[B]} \\
\vdots & \vdots & \vdots & \vdots \\
\frac{A \quad \stackrel{1}{A}}{A \oplus B} \oplus I_{1} & \frac{B \quad \dot{\perp}}{A \oplus B} \oplus I_{2} & \frac{A \oplus B}{B} & \dot{A}
\end{array} \oplus E
$$

In this case, $\oplus$ cannot be used to express $\neg$. It is thus not clear whether a complete system can be obtained without adding $\neg$ as a primitive together with the full classical set of rules for it.

Finally, the same method can be applied to Schönfinkel's (1924) quantifier $\left.\right|^{x}$, the basis of his $U$ combinator. $A(x) \mid{ }^{x} B(x)$ is true iff for every $x$, not both $A(x)$ and $B(x)$ hold. Sound and complete sequent calculus rules are

$$
\frac{A(a), B(a), \Gamma \vdash \Delta}{\Gamma \vdash \Delta, A(x) \mid{ }^{x} B(x)}\left|{ }^{x} R \quad \frac{\Gamma \vdash \Delta, A(t) \quad \Gamma \vdash \Delta, B(t)}{A(x) \mid{ }^{x} B(x), \Gamma \vdash \Delta}\right|{ }^{x} L
$$

which lead to the following intelim rules,

$$
\begin{aligned}
& {[A(a)] \quad[B(a)]} \\
& \vdots \\
& \frac{\dot{\perp}}{A(x) \mid{ }^{x} B(x)} \mid{ }^{x} I \quad \frac{A(a) \mid{ }^{x} B(a) \quad A(t) \quad B(t)}{\perp}
\end{aligned}
$$

all of course with the usual eigenvariable conditions on $a$. 


\section{Conclusion}

The specific question of how to come up with left and right rules for the Sheffer stroke in the style of $\mathbf{L K}$, or introduction and elimination rules in the style of NK might seem like a mere recreational logic puzzle. Nevertheless, the considerations above have highlighted some interesting aspects of sequent calculus and natural deduction. The first is that there are general methods which generate such rules automatically from their truth tables. Not only are the resulting systems complete, they also enjoy the same properties (like cut-elimination) as the standard systems, and transformations from one system to another (such as the translations between proofs and derivations sketched in Proposition 3) work in both multiple-conclusion (classical) and single-conclusion (intuitionistic) systems. Our consideration of how to make a single-conclusion natural deduction calculus classical revealed the importance of the $\perp_{C}$ rule in this respect: $\neg L$ allows us to move formulas from the succedent of a sequent to the right (where they then appear negated), and and $\neg E$ allows us in effect to turn a derivation of $A$ into one of $\perp$ from $\neg A$. The rules $\mid L_{C}$ (of the proof of Proposition 5) and $\perp_{C}$ enable us to reverse this, i.e., to move negated formulas from the antecedent of a sequent to the succedent, and to transform a derivation in which $\neg A$ is an open assumption into one with $A$ instead as the conclusion. This means that the restriction to a single formula on the right, which corresponds to the restriction to single conclusions in natural deduction, can be circumvented. The same trick can be used whenever the new connective(s) define(s) $\neg$ (as in the case of $\mid$ ), but it can also be used to show how a pair of intelim rules can be made classically complete by adding rules for $\neg$ including the $\perp_{C}$ rule.

Acknowledgements I am grateful to Allen Hazen and Jeff Pelletier for helpful comments as well as the question which originally prompted this paper.

\section{References}

Baaz M, Fermüller CG (1996) Intuitionistic counterparts of finitely-valued logics. In: Proc. 26rd International Symposium on Multiple-valued Logic, IEEE Press, Los Alamitos, pp 136-141, DOI 10.1109/ISMVL. 1996.508349, URL http://dx.doi.org/10.1109/ISMVL . 1996.508349

Baaz M, Fermüller CG, Zach R (1993) Systematic construction of natural deduction systems for many-valued logics. In: Proc. 23rd International Symposium on Multiple-valued Logic, IEEE Press, Los Alamitos, pp 208-213, DOI 10.1109/ISMVL.1993.289558, URL http://dx.doi.org/10.1109/ISMVL. 1993. 289558

Baaz M, Fermüller CG, Zach R (1994) Elimination of cuts in first-order finite-valued logics. Journal of Information Processing and Cybernetics EIK 29(6):333-355, URL http://ucalgary.ca/rzach/ papers/mvlcutel.html

Fitch F (1952) Symbolic Logic: An Introduction. Ronald Press, New York

Gentzen G (1934) Untersuchungen über das logische Schließen I-II. Math Z 39:176-210, 405-431

Harrop R (1960) Concerning formulas of the types $A \rightarrow B \vee C, A \rightarrow(E x) B(x)$. Journal of Symbolic Logic 25(1):27-32, URL http://www.jstor.org/stable/2964334

Hazen AP, Pelletier FJ (2014) Gentzen and Jaśkowski natural deduction: Fundamentally similar but importantly different. Studia Logica 102(6):1103-1142, DOI 10.1007/s11225-014-9564-1, URL http: //dx.doi.org/10.1007/s11225-014-9564-1

van Heijenoort J (ed) (1967) From Frege to Gödel. A Source Book in Mathematical Logic, 1879-1931. Harvard University Press, Cambridge, MA

Jaśkowski S (1934) On the rules of suppositions in formal logic. No. 1 in Studia Logica, Seminarjum Filozoficzne. Wydz. Matematyczno-Przyrodniczy UW, Warsaw, reprinted in (McCall 1967, 232-258)

Lemmon EJ (1965) Beginning Logic. Nelson, London

McCall S (ed) (1967) Polish Logic 1920-1939. Oxford University Press, London 
Parigot M (1992a) Free deduction: An analysis of "computations" in classical logic. In: Voronkov A (ed) Logic Programming, Springer Berlin Heidelberg, Lecture Notes in Computer Science, vol 592, pp 361380, DOI 10.1007/3-540-55460-2_27, URL http://dx.doi.org/10.1007/3-540-55460-2_27

Parigot M (1992b) $\lambda \mu$-Calculus: an algorithmic interpretation of classical natural deduction. In: Voronkov A (ed) Logic Programming and Automated Reasoning. Proceedings LPAR'92, Springer, Berlin, LNAI 624, pp 190-201, URL http://dx.doi.org/10.1007/BFb0013061

von Plato J (2001) Natural deduction with general elimination rules. Archive for Mathematical Logic 40(7):541-567, DOI 10.1007/s001530100091, URL http://dx.doi.org/10.1007/ s001530100091

Prawitz D (1965) Natural Deduction. Stockholm Studies in Philosophy 3, Almqvist \& Wiksell, Stockholm

Price R (1961) The stroke function and natural deduction. Zeitschrift für mathematische Logik und Grundlagen der Mathematik 7:117-123

Read S (1999) Sheffer's stroke: a study in proof-theoretic harmony. Danish Yearbook of Philosophy 34:7-23

Schönfinkel M (1924) Über die Bausteine der mathematischen Logik. Mathematische Annalen 92:305-316, English translation in (van Heijenoort 1967, pp. 355-366)

Suppes P (1957) Introduction to Logic. Van Nostrand Reinhold, New York

Takeuti G (1987) Proof Theory, 2nd edn. Studies in Logic 81, North-Holland, Amsterdam

Troelstra AS, Schwichtenberg H (2000) Basic Proof Theory, 2nd edn. Cambridge University Press, Cambridge

Zach R (1993) Proof theory of finite-valued logics. Diplomarbeit, Technische Universität Wien, Vienna, Austria, URL http://ucalgary.ca/rzach/papers/ptmvl.html

\section{Errata}

The $\mid E$ rule of section 7 should read:

$$
\triangleright \begin{array}{ll}
A \mid B & \\
A & \\
B & \\
\perp & \mid E
\end{array}
$$

The rule printed in the text corresponds instead to a special version of the $\| E^{\prime}$ rule of section 4 . However, that rule is also incorrect. The $\| E^{\prime}$ rule corresponding to $\| L^{\prime \prime}$ is

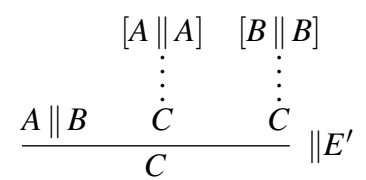

